Case Report

\title{
Association of Acute Myeloid Leukemia and Systemic Lupus Erythematosus: A Case Report
}

\author{
Romaric Mahutondji Massi, ${ }^{1,}$, Mouna Lamchahab ${ }^{1}$, Marième Camara ${ }^{2}$, Bienvenu Houssou ${ }^{1}$, \\ Bouchra Oukkache ${ }^{2}$, Asmaa Quessar ${ }^{1}$ \\ ${ }^{1}$ Department of Haematology and Paediatric Oncology, Faculty of Medecine and Pharmacy, Hassan II University, Casablanca, Morocco \\ ${ }^{2}$ Departement of Hematology Laboratory, Faculty of Medecine and Pharmacy, Hassan II University, Casablanca, Morocco
}

Email address:

massiroswork@yahoo.fr (R. M. Massi),mlamchahab@hotmail.fr(M.Lamchahab),macodou89@outlook.fr(M. Camara), houbien85@yahoo.fr(B.Houssou), bouchraoukkache@gmail.com(B. Oukkache),quessar.a@gmail.com(A. Quessar)

${ }^{*}$ Corresponding author

\section{To cite this article:}

Romaric Mahutondji Massi, Mouna Lamchahab, Marième Camara, Bienvenu Houssou, Bouchra Oukkache, Asmaa Quessar. Association of Acute MyeloidLeukemia andSystemic Lupus Erythematosus: A CaseReport. American Journal of Internal Medicine.

Vol. 6, No. 1, 2018, pp. 25-28.doi: 10.11648/j.ajim.20180601.14

Received: March 2, 2018; Accepted: March 14, 2018; Published: April 8, 2018

\begin{abstract}
Systemic lupus erythematosus is an autoimmune Inflammatory disease that can affect multiple systems and organs of the body including skin, kidneys, lungs, joints and nervous system.Some previous studies up light thatSLE was associated with increased risk of Acute leukemia (SIR = 2.3). The incidence ofthe association of AMLand SLE is not known but there are few case reports in literature. The aim of this report was to up light the difficulties wich occurred in the diagnosis and the management of acute myeloid leukemia in patient with systemic lupus erythematosus. Patient, 43 years old, diabetic treat withinsulin, wichsister is followed for Behcet's disease, was admited for AML with trisomy of chromosome 4 and 8 and thrombophlebitis of the superior sagittal sinus, Chest CT-Scan showed minimal bilateral pleural effusion. She was treated according to Morocco National protocol AML-MA-2011. CT-Scan was done atday 12 ofchemotherapy and noticedthe persistance of the minimal bilateral pleural effusion. At day 19 the patient presentedmalar rash, and right axillary adenitis. The biopsy of the adenitis show the presence of LE cells. According to internal physician recommendations we add steroids to the treatement. The cough and fever deasapear. The CT-scan for control was normal after two weeks of steroids. Patient is in complete remission after induction I. After a following-up of five months, the patient still well,but present severy infections during chemotherapy cycles and a bad tolerance for the treatement. The association SLE-AML is rare. The diagnosis of the association SLE-AML is difficult. It is management is also difficult according to commorbidity, severy infectious because of dicline of immunity, and less tolerance to AML chemotherapy.
\end{abstract}

Keywords: Acute Myeloid Leukemia, Systemic Lupus Erythematosus, Association

\section{Introduction}

Systemic lupus erythematosus (SLE) is an autoimmune Inflammatory disease that can affect multiple systems and organs of the body including skin, kidneys, lungs, joints and nervous system. Some previous studies up light that, SLE increase the risk of Hematologic malignancies wichare definiteasa heterogeneous group of diseases characterized by the abnormal (malignant) growth and/or accumulation of hematopoietic cells in the blood, bone marrow and/or lymph nodes.

In 2005, ameta-analysis conducted by Zintzaras and al,had shown that there was a moderate risk of lymphoma incidence in patients with SLE with an estimated standardized incidence ratio (SIR) 7-times higher compared with the general population [1]. In 2014, in ameta-analysis, including 401 cases of hematologic malignancies identified in a total cohort of 67,929 individuals with a diagnosis of SLE, Emmanuel Apor and al had shown thatSLE was associated with increased SIR of Acute leukemia (SIR = 2.3) [2]. The incidence ofthe 
association of Acute Myeloid Leukemiaand SLE is not known but there are few case reports in literature. The firstcase had been reported by Lee in1955[3].

This is a reportof a case of systemic lupus erythematosus associated with Acute Myeloid Leukemia in an young woman.

\section{Case Presentation}

Patient, 43 years old, mother of 02 children, diabetic treat withinsulin, wichsister is followed for Behcet's disease,present one month before the first consultation, asthenia, cutaneous pallor, rebels headache, multiple arthralgia, cough and fever.The initial clinical examination had noticed a patient with Performans status (WHO) at 2, febrile at $39^{\circ} \mathrm{Celsius}$, blood preasure was at $110 / 80 \mathrm{mmHg}$, respiratory rate was at 20 cycles/min. Heart and lung auscultation was normal. No hemorrhagic syndroma, no lymph nodes, no splenomegalia, no hepatomegalia.CBC showed hemoglobin level at $7.8 \mathrm{~g} / \mathrm{dl}$, white blood cells was at $2430 / \mathrm{ml}$ with $54 \%$ blasts, platelet was at $56000 / \mathrm{ml}$. The bone marrow aspiration and immunophenotyping conclued to AML 2. The karyotype found trisomy of chromosome 4 and 8 . The viral serology were negative. Tuberculosis PCR and Galactomanann antigenemia was negative. Blood cultures were negative. The research for lupus antibodies, nuclear and DNA antibodies were negative. MRI found thrombophlebitis of the superior sagittal sinus(Figure 1). Chest CT-Scan showed minimal bilateral pleural effusion.
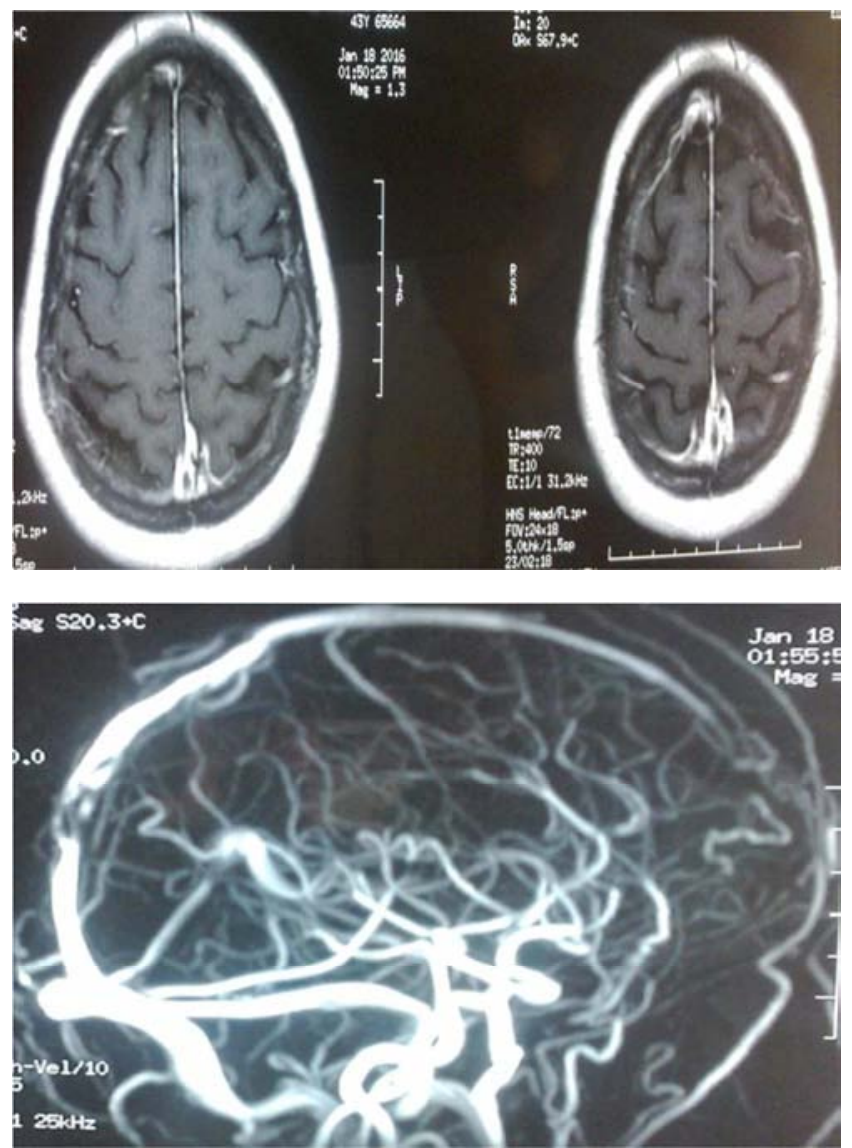

Figure 1. Thrombophlebitis of the superior sagittal sinus.
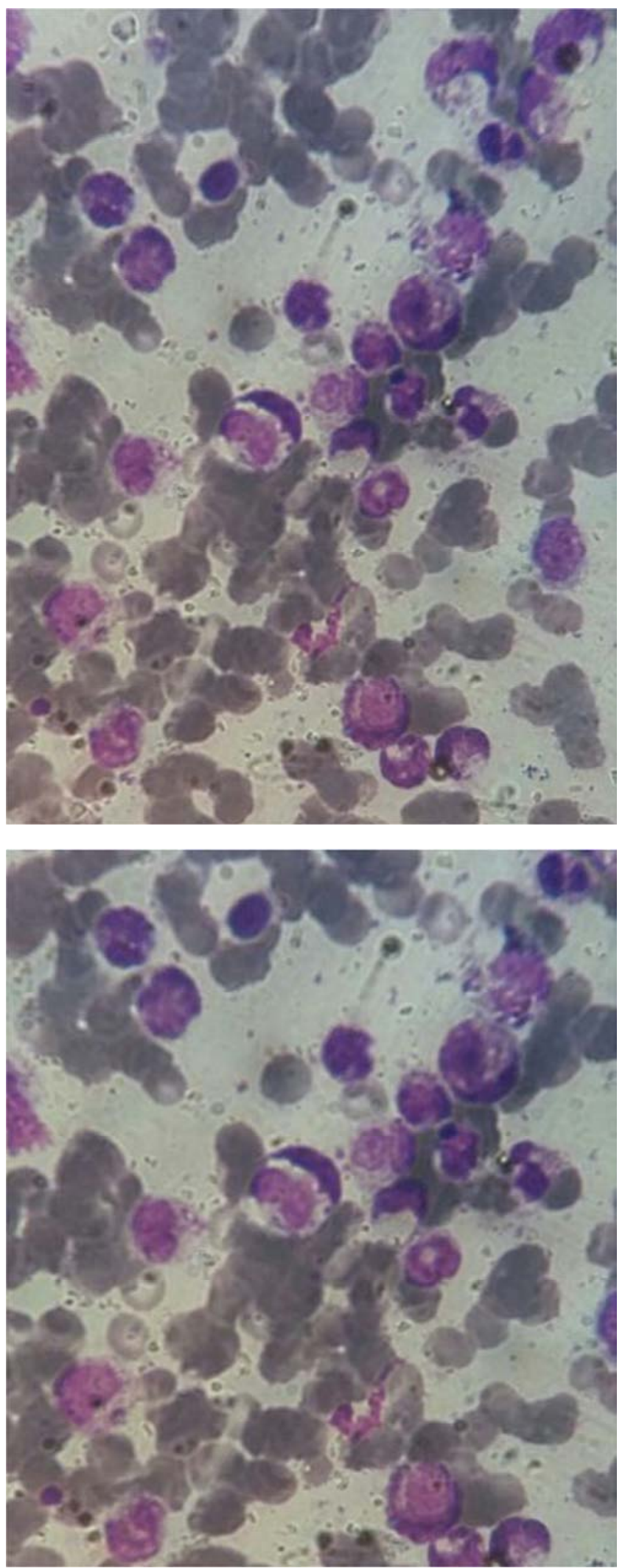

Figure 2. LE Cells.

A multidisciplinary consensuel meeting of hematologists, pulmonologists, neurologists decided to treat the patient according to protocol AML-MA-2011, recommand toadministrate $0.6 \mathrm{ml}$ of enoxaparintwice a day,to maintain by 
itterative transfusion,platelet rate $\geq 30.000 / \mathrm{mL}$ because of the use of enoxaparin, and hemoglobin level $\geq 10 \mathrm{~g} / \mathrm{dl}$ because of the rebels headeache. The evolution was marked by a significant decline of headache but a persistent fever and coughdespite the administration of multiple drugs (Ceftazidim, Amikacin, Imipenem, Ciprofloxacin, Voriconazol, Cicloviral) AnotherCT-Scan was done atday 12 ofchemotherapy and noticedthe persistance of the minimal bilateral pleural effusion.At day 19 the patient presentedmalar rash, and rightaxillary adenitis. The biopsy of the adenitis show the presence of LE cells(Figure 2). To summary it is a patient treat for AML wich present also some ACR criteria for the diagnosis of SLE: malar rash, arthitis, pleural effusion,thrombopenia less than $100.000 / \mathrm{ml}$, leucopenia and LE cells.According to internal physician recommendationssteroids was added to the treatement. The cough and fever deasapear. The CT-scan for control was normal after two weeks of steroids. Patient is in complete remission after induction I.After a following-up of five months, the patient still well,but present severy infections during chemotherapy cycles and a bad tolerance for the treatement.

\section{Discusssions}

Cancer risk in SLE is a topic of increasing interest, but to date much of the focus has been directed at lymphoma, especially Non-Hodgkin's lymphoma. The studies on the association AML-SLE are rare. In 2013, Mary Lu and alin a cohort of 16, 409 patients with SLE observed33 case ofnon-lymphoma hematological malignancies [4]. Of these 33 non-lymphoma cases, 13 were of lymphoid lineage: multiple myeloma $(\mathrm{N}=5)$, plasmacytoma $(\mathrm{N}=3)$, B-cell chronic lymphocytic leukemia, B-CLL $(\mathrm{N}=3)$, precursor cell lymphoblastic leukemia $(\mathrm{N}=1)$, and unspecified lymphoid leukemia $(\mathrm{N}=1)$.

The remaining 20 cases were of myeloid lineage: myelodysplastic syndrome, MDS $(\mathrm{N}=7)$, acute myeloid leukemia, AML $(\mathrm{N}=7)$, chronic myeloid leukemia, $\mathrm{CML}$ $(\mathrm{N}=2)$, and 4 unspecified leukemias. This studie up light that the association SLE-AML is very rare because only seven cases of AML were diagnosed in 16,409 patients with SLE. Most of the hematological malignanciesdeveloped in SLEwere female, which reflected the fact that $90 \%$ of SLE patient are female [5]. That is also the same think in this report.

In 2009, Bjorn L and al conducted an meta-analysis completed by a nested case control study based on Swedish registers among 6438 patients with SLE. They identified eigth cases of AML in SLE but only three were female.In female SLE cases with SLE, the median age at AML diagnosis was 48 years (range $34-72$ ) [6]. That is also what was noticed in this case. The case of this reporthad43 years old.All the cases of AML in SLE were diagnosed during the following of SLE. In Bjorn L and alcohort, the median of following-up before the diagnosis was 77 months [29-110 months].

The difficuly in this case, is that the patient was not followed for SLE before the diagnosis of AML. She had some SLE manifestations (Pleuritis, arthritis, thrombopenia) but this signs are not specifics or SLE and can be present in AML also. Its the persitence of symptomatology despite all drugs wich were administrated, the apparition of malar rash and the identification of LE cells wich permit forto update the diagnosis. So, whensteroids were administratedall clinical and radiological signs deasapear and confirm the diagnosis.

The presence of haematological abnormalities, anaemia, leucopenia and to a lesser extent thrombocytopenia are common clinical manifestations of the SLE disease, often independent of disease severity and can be present also in AML.

So it is necessarythat SLE patients beensubject to regular check-ups for clinical manifestations andlaboratory tests, to showif apossible transition had occurred from cytopenias via MDS to AML. Interestingly, leucopenia was the only clinical SLE-associated finding with a significantly elevated OR for leukaemia development.

The frequency of a preceding myelodysplastic phase before leukaemia was at least comparable with the estimated $25 \%$ that has been observed in the general leukaemia population [7]. Some previously known associations between rheumatic diseases and leukaemia do exist. In a population-based cohort of Wegener's granulomatosis, a more than 5-fold increased risk of leukaemia was found [8] [9].

The relation between SLE and leukemia is rather unclear. One could hypothesize that some of the drugs used to treat SLE, such as the alkylator cyclophosphamide among others, can increase the risk of developing myeloid neoplasms such as myelodysplastic syndrome (a pre-leukemic state) and acute myeloid leukemia [10] [11].

So chemotherapeutic drugs, constitute one of the relatively few known aetiological risk factors for leukaemia. Azacytidin is an anti-metabolite that has been used in SLE treatment since the 1960s both for treating disease manifestations and as a glucocorticoid-sparing drug. It could induce defective DNA mismatch repair, possibly promoting survival of cells for a leukaemic clone [12]. On the contrary, anti-malarial drugs like chloroquine, are immune modulating drugs often used in patients with SLE that have recently been reported to exert anti-neoplastic properties. They are strongly DNA intercalating, preventing mutations in cells with a high mitotic rate and improving cellular mechanisms of DNA repair after the damage was caused by alkylating therapy [13]. This effect of anti-malarial drugs can explain why AML are rare in SLE patients.

The therapeutic management of AML in SLE is difficult because of commorbidity(kidneys, lungs, nervous system diseases), severy infectious because of dicline of immunity, and less tolerance to AML chemotherapy. In fact febrile neutropenia is the most frequent and important complications of chemotherapyduring the management of acute myeloid leukemia. It is generally the first factor of mortality. It incidence had been estimated between $70-100 \%$ during the neutropenic phase after intensive chemotherapy.Immune deficiency developedduring malignancy and chemotherapy is 
the first factor wich explain febrile neutropenia in acute myeloid leukemia patient. Because of this immune deficiency serious infection will manifest in neutropenic patient with minimal symptoms. In AML patient with SLE this immune deficiency is more important and patient present graveous infections during neutropenia. It what was noticed also when managed this patient wich present several infection during a different phase of chemotherapy.

The median survival time after the diagnosis of AML in SLE patients is 7 months (2-18 months) [14] [15]. In our case, after a following-up of five months, the patient still well,but present severy infections during chemotherapy cycles and a bad tolerance for the treatement.

\section{Conclusion}

The association SLE-AML is rare.The presence of haematological abnormalities, are common clinical manifestations of the SLE disease, often independent of disease severity and can be present also in AML. So the diagnosis of the association SLE-AML is difficult. It is management is also difficult according tocommorbidity, severy infectious because of dicline of immunity, and less tolerance to AML chemotherapy.

\section{Abbreviations}

Systemic lupus erythematosus (SLE); Acute Myeloid Leukemia (AML).

\section{Authors' Contributions}

RM and MCprepared the draft and BH, ML, BO, and AQ all reviewed and contributed to the final manuscript.

\section{Conflict of Interest}

The authors declare that they have no competing interests.

\section{Consent for Publication}

Written consent to publish this report was obtained from the patient.

\section{References}

[1] Zintzaras E, Voulgarelis M, Moutsopoulos HM. The risk of lymphoma development in autoimmune diseases: a meta-analysis. Arch Intern Med 2005;165:2337-44.
[2] Emmanuel Apor,Jennifer O'Brien, Merin Stephen, Jorge J. Castillo d. Systemic lupus erythematosus is associated with increased incidence of hematologic malignancies: A meta-analysis of prospective cohort studies. Leukemia Research 38 (2014) 1067-1071.

[3] Lee SL. Clinical experience with the LE cell test. J Mount Sinai Hosp NY 1955;22:74-8.

[4] Mary Lu, Sasha Bernatsky, Rosalind Ramsey-Goldman, Michelle Petri, Susan Manzi, Murray B and al. Non-Lymphoma Hematological Malignancies in Systemic Lupus Erythematosus. Oncology. 2013; 85(4).

[5] Kang KY, Kim HO, Yoon HS, et al. Incidence of cancer among female patients with systemic lupus erythematosus in Korea. Clin Rheumatol 2010;29: 381-8.

[6] Bjorn Lofstrom, Carin Backlin, Christer Sundstrom, Eva Hellstrom-Lindberg, Anders Ekbom, Ingrid E. Lundberg. Myeloid leukaemia in systemic lupus erythematosus-a nested case-control study based on Swedish registers. Rheumatology 2009;48:1222-1226.

[7] Juliuson G, Antunovic P, Derolf A et al. Real world data on decision to treat and outcomes from the Swedish acute Leukemia Registry. 2009 113: 4179-4187.

[8] Knight A, Askling J, Ekbom A. Cancer incidence in a population-based cohort of patients with Wegener's Granulomatosis. Int J Cancer 2002;100:82-85.

[9] Knight A, Askling J, Granath F, Sparen P, Ekbom A. Urinary bladder cancer in Wegener's granulomatosis: risks and relation to cyclophosphamide. Ann Rheum Dis 2004;63:1307-11.

[10] Bhatia S. Therapy-related myelodysplasia and acute myeloid leukemia. Semin Oncol 2013;40:666-75.

[11] Bernatsky S, Joseph L, Boivin JF, et al. The relationship between cancer and medication exposures in systemic lupus erythaematosus: a case-cohort study. Ann Rheum Dis 2008;67:74-9.

[12] Leone G, Pagano L, Ben-Yehuda D, Voso MT. Therapy-related leukaemia and myelodysplasia: susceptibility and incidence. Haematologica 2007;92:1389-98.

[13] Ruiz-Irastorza G, Ugarte A, Egurbide MV et al. Antimalarials may influence the risk of malignancy in systemic lupus erythematosus. Ann Rheum Dis 2007;66:815-7.

[14] Kwong YL, Au WY, Liang RHS. Acute myeloid leukemia after azathioprine treatment for autoimmune diseases: association with -7/7q-. Cancer Genet Cytogenet 1998;103:94-7.

[15] Leone G, Pagano L, Ben-Yehuda D, Voso MT. Therapy-related leukaemia and myelodysplasia: susceptibility and incidence. Haematologica 2007;92:1389-98. 